\title{
A remarkable correlation between short period gravity waves and semiannual oscillation of the zonal wind in the equatorial mesopause region
}

\author{
N. Venkateswara Rao ${ }^{1}$, T. Tsuda ${ }^{1}$, and Y. Kawatani ${ }^{2}$ \\ ${ }^{1}$ Research Institute for Sustainable Humanosphere, Kyoto University, Uji, Japan \\ ${ }^{2}$ Japan Agency for Marine-Earth Science and Technology, Yokohama, Japan \\ Correspondence to: N. Venkateswara Rao (ionovenki@gmail.com) \\ Received: 10 January 2012 - Accepted: 5 April 2012 - Published: 19 April 2012
}

\begin{abstract}
The variability of zonal winds and the horizontal wind velocity variance of short period (20-120 min) gravity waves (GWs) in the equatorial mesopause region are studied using medium frequency (MF) radar observations from Pameungpeuk $\left(7.4^{\circ} \mathrm{S}, 107.4^{\circ} \mathrm{E}\right)$ during 2004-2010. The zonal winds display a distinct semiannual oscillation (called mesospheric semiannual oscillation, MSAO), with westward winds during equinoxes and eastward winds during solstices. Furthermore, the westward winds during March equinox are larger during 2008 and 2009. The short period GW variance also shows a semiannual oscillation with enhanced activity during equinoxes. A good correlation is observed between the zonal winds and the short period GW variance from 2008-2010, with the winds being westward during the times of enhanced GW activity. Such a correlation, however, is less obvious during 2004-2006. The long period (10-20 h) GW variance, on the other hand, does not show such a correlation throughout the observation period.
\end{abstract}

Keywords. Meteorology and atmospheric dynamics (Middle atmosphere dynamics; Waves and tides) - Radio science (Remote sensing)

\section{Introduction}

Gravity waves (GWs) generated in the lower atmosphere propagate upward and reach significant amplitudes by the time they reach the mesosphere and lower thermosphere (MLT) region. Consequently, they break/dissipate due to convective and dynamical instabilities, resulting in acceleration of the background wind and modification of the ther- mal structure and general circulation in that region (Fritts and Alexander, 2003). Gravity waves can further propagate into the ionosphere and play an important role in the formation of quasi-periodic echoes in the E region (e.g. Venkateswara Rao et al., 2008) and equatorial plasma irregularities in the $F$ region (e.g. Taori et al., 2011). The upward propagating gravity waves, before reaching the MLT region, undergo critical level interaction with background winds at various levels of the atmosphere, depending on the wave phase velocity relative to the background winds, such as the Tropical Easterly Jet (TEJ), quasi-biennial oscillation (QBO) and stratopause semiannual oscillation (SSAO) in the stratosphere.

Zonal-mean zonal winds in the tropical mesosphere exhibit a prominent semiannual oscillation, with westward winds during equinoxes and eastward winds during solstices, called the mesospheric semiannual oscillation (MSAO) (Hirota, 1978). The MSAO has been studied through various ground and satellite-based wind and temperature measurements (Burrage et al., 1996; Garcia et al., 1997; Sridharan et al., 2007). These studies have shown that the westward phase of the MSAO is symmetric over the equator and the westward wind during the March equinox undergoes large inter-annual variability. The MSAO is considered to be wave-driven by model studies, including the effects of small-scale gravity waves, intermediate-scale Kelvin and inertia-gravity waves, diurnal tide deposition, as well as forcing due to meridional advection (Dunkerton, 1982; Lieberman and Hays, 1994; Sassi and Garcia, 1997; Richter and Garcia, 2006).

Gurubaran and Rajaram (2001) studied the mean winds, tides and GWs over Tirunelveli $\left(8.7^{\circ} \mathrm{N}, 77.8^{\circ} \mathrm{E}\right)$ and showed that short-term tidal variability through GW-tidal interaction 
contributes to the variation of the strength of the time-mean westward flow. There also exist some long-term studies on the relation between the zonal winds and GW activity (Offermann et al., 2011; Hoffmann et al., 2011). A study by Hoffmann et al. (2011) shows that the trends in zonal wind at $75 \mathrm{~km}$ correlates with that of GW activity at altitudes above $80 \mathrm{~km}$.

Different models consider different sources as drivers and tune to reproduce the observed MSAO; yet, the driving sources of this oscillation are not well-understood. Currently, there is considerable ambiguity on the driving sources of the MSAO. In this context, the present results will help in better understanding the MSAO and its relation to GWs.

\section{Data}

The MLT wind data used in this study were acquired using a medium frequency (MF) radar located at Pameungpeuk $\left(7.4^{\circ} \mathrm{S}, 107.4^{\circ} \mathrm{E}\right)$, Indonesia (Venkateswara Rao et al., $2011 \mathrm{a}, \mathrm{b}$ ). This radar operates at a frequency of $2.008 \mathrm{MHz}$ with a peak power of $30 \mathrm{~kW}$. The antenna array, consisting of three antennas arranged in an equilateral triangle, is used for both transmission and reception. This radar measures winds using the spaced antenna technique, in the height range of $78-98 \mathrm{~km}$, with resolutions of $2 \mathrm{~km}$ in height and $2 \mathrm{~min}$ in time. The data acceptance rate is highest at $\sim 88 \mathrm{~km}$. In the present study, we use the Pameungpeuk MF radar data collected from April 2004 to December 2010. The radar was not in operation during 10 August 2004 to 6 November 2004, 7 28 July 2005, and 13 February 2007 to 2 October 2007. During 16 August 2006 to 16 January 2007, the data are sparse and hence not used for the present study.

The method of GW variance calculation is as follows. High resolution (1-2 min) horizontal wind data acquired by the MF radar are averaged to get $10 \mathrm{~min}$ interval data. Short data gaps in the $10 \mathrm{~min}$ time series were linearly interpolated. From this dataset, contributions from diurnal, semi-, ter-, and quarter-diurnal tides are removed (Venkateswara Rao et al., 2011b). Then, GW variance is calculated for three bands of periods (20-120 min, 2-10 h, 10-20 h). For calculating the GW variance in the short period band (20-120 min), a block of three hours (which contains 18 data points), sliding forward by one hour, is subjected to Butterworth high-pass filter (of order 5) with a cut off of $120 \mathrm{~min}$. Variance is then calculated from this filtered output and attributed to the middle of each three-hour interval. In this manner, we calculate short period GW variance for $24 \mathrm{~h}$. Daily variance is calculated by averaging the hourly variances. For calculating the $\mathrm{GW}$ variance in $2-10 \mathrm{~h}$ and $10-20 \mathrm{~h}$ period bands, we use a data block of two days and subjected to band pass filter.

\section{Results}

Six-year (2004-2006 and 2008-2010) composites (average at each month of all years) of monthly-averaged mean winds (top panel), GW variance in the $20-120 \mathrm{~min}$ band (second panel), in the $2-10 \mathrm{~h}$ band (third panel), and in the $10-20 \mathrm{~h}$ band (fourth panel) are shown in Fig. 1a and $b$ for the zonal and the meridional components, respectively. The white space in Fig. 1a and b represents the data gaps due to lack of a sufficient number of data points to compute the six-year composite of GW variance. Accordingly, we will not discuss the winds and short period GW variance at higher altitudes and thus confine to altitudes $<\sim 94 \mathrm{~km}$. The zonal winds in Fig. 1a display a distinct semiannual oscillation, with westward winds during equinoxes and eastward winds during solstices. Furthermore, the peak westward winds around March equinox are larger than those around September equinox. The meridional winds in Fig. 1b show a clear annual oscillation (AO), with southward winds from the middle of March to October and northward winds for the rest of the time.

The 20-120 min GW variance (second panel) in the zonal wind shows a semiannual oscillation, with primary enhancement during March equinox and secondary enhancement during September equinox. The $20-120 \mathrm{~min}$ GW variance in the meridional component also shows a similar variability, with the meridional variance being larger than the zonal component above $\sim 92-93 \mathrm{~km}$. The zonal component of GW variance in $2-10 \mathrm{~h}$ band shows some seasonal variation, with less variance during the middle of March to July, compared to rest of the year. Largest variance is observed between January and March. The meridional component also shows similar variation, except that the enhanced GW activity between January and March is little larger than that in the zonal component. The $\mathrm{GW}$ variance is also larger between July and December. The GW variance in $10-20 \mathrm{~h}$ band also shows similar seasonal variation to that of $2-10 \mathrm{~h}$ band, with enhanced variance during January-February in the zonal component and during January-March and JulyDecember (changes with height) in the meridional component.

The zonal wind shows predominantly the SAO and the meridional wind shows the AO. Furthermore, the short period $\mathrm{GW}$ variance also shows the $\mathrm{SAO}$, similar to zonal wind. Since the zonal wind and short period GW variance have similar seasonal variation, we will focus on the relation between the two in the present study, neglecting the meridional wind. Further, since the GW variance in each band is nearly similar in both the zonal and the meridional components, we will compute a total $\mathrm{GW}$ variance in each band, by averaging the $\mathrm{GW}$ variance in the two components $\left(u^{\prime 2}+v^{\prime 2}\right) / 2$.

Figure 2 a shows the monthly mean prevailing zonal winds from January 2004 to December 2010 at heights of $88 \mathrm{~km}$ and $92 \mathrm{~km}$, while the monthly mean total GW variance $\left(u^{\prime 2}+v^{\prime 2}\right) / 2$ with wave period in the band of 20-120 min, 2$10 \mathrm{~h}$ and $10-20 \mathrm{~h}$ are shown in Fig. 2b, c and d, respectively. 
(a) Zonal
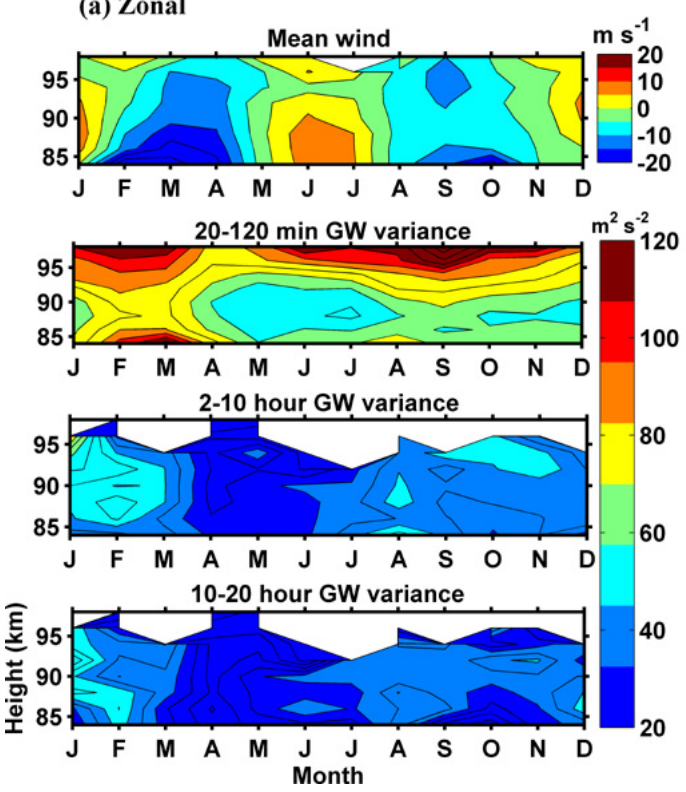

(b) Meridional
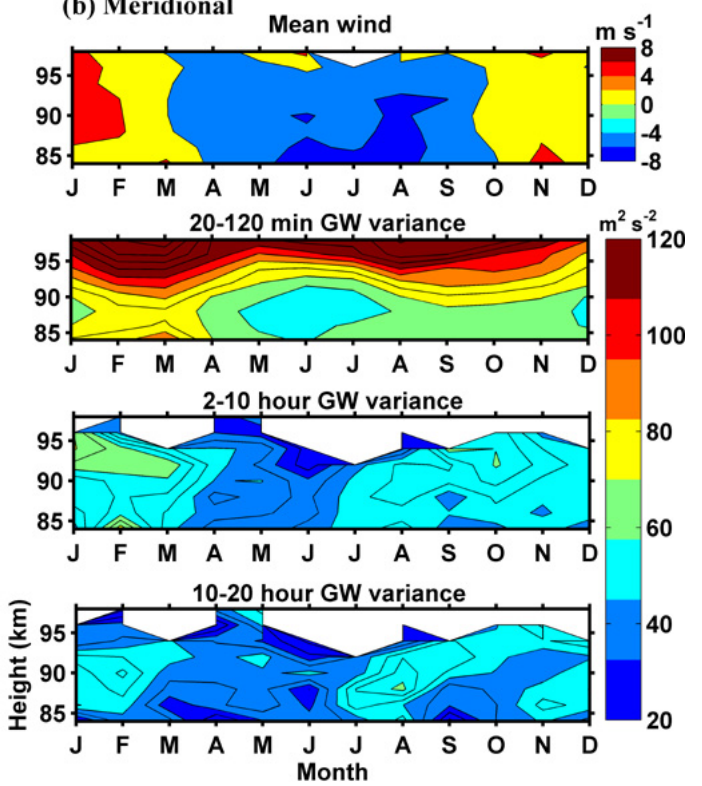

Fig. 1. A six-year (2004-2006 and 2008-2010) composite of monthly- averaged mean winds (top panel), GW variance in the 20-120 min band (second panel), in the $2-10 \mathrm{~h}$ band (third panel), and in the 10-20 h band (fourth panel) for (a) zonal and (b) meridional components, respectively.

Note that, to increase clarity, the values at $92 \mathrm{~km}$ are shifted forward by 0.5 months. The vertical lines at each data point represent standard deviations for the monthly mean values. The zonal winds in Fig. 2a display a clear semiannual oscillation in all years, with westward winds during equinoxes and eastward winds during solstices. Further, in each year the first westward cycle of the oscillation is generally stronger than the second one, except during 2010 where amplitudes of both the cycles are nearly equal. For eastward winds, both cycles have nearly equal amplitudes throughout the observation period. While the peak westward winds are stronger than the peak eastward winds in 2008 and 2009, they are nearly equal in 2005 and 2010. In 2004, 2006 and 2007, the data are not sufficient to bring out this kind of variability. The standard deviation in monthly mean zonal winds is generally $4-10 \mathrm{~m} \mathrm{~s}^{-1}$ and with a maximum of $20 \mathrm{~m} \mathrm{~s}^{-1}$.

The winds during 2008 show an anomalous behavior, being westward for most of the time. Thus, the annual mean zonal wind during 2008 is more negative $\left(-7.25 \mathrm{~m} \mathrm{~s}^{-1}\right)$ than during $2009\left(-0.79 \mathrm{~m} \mathrm{~s}^{-1}\right)$ and $2010\left(-3.73 \mathrm{~m} \mathrm{~s}^{-1}\right)$. In 2005 also, the annual mean winds are westward $\left(-4.2 \mathrm{~m} \mathrm{~s}^{-1}\right)$. Although the semiannual oscillation is clearly observed during all three years, in 2008 the winds remain westward, despite the SAO.

Monthly mean short period (20-120 min) GW variance, shown in Fig. 2b, is less during 2004-2005 than in 2006 and 2008-2010. Further, during the 2008-2010 period, the GW variance shows distinct semiannual variation, with enhanced variance during equinoxes. From 2008 to 2009 the GW variance shows an equinoxial asymmetry, with a larger variance in February-March than in September-October. In 2010, however, the variance during September-October is slightly larger than during February-March. The large data gaps in 2004, 2006, and 2007 preclude comments about the semiannual variability of GW activity in these years. The monthly mean errors are, in general, in the range of $20-60 \mathrm{~m}^{2} \mathrm{~s}^{-2}$.

Figures $2 \mathrm{c}$ and $\mathrm{d}$ correspond to $\mathrm{GW}$ variance in $2-10 \mathrm{~h}$ and $10-20 \mathrm{~h}$ bands. The $\mathrm{GW}$ variance in $2-10 \mathrm{~h}$ band shows some enhancement in March equinox in 2006, and between 2008 and 2010 . In the $2-10 \mathrm{~h}$ band, however, the seasonal variation is less systematic, compared to the short period GW variances in Fig. 2b, with no enhancement in SeptemberOctober. The GW variance in the $10-20 \mathrm{~h}$ band shows enhancement during January 2006, January and August 2009, and July, February, August and December 2010. Although the total variance is shown here, individual variances of zonal and meridional components also show nearly similar behavior, as illustrated in Fig. 1.

The most interesting aspect in Fig. 2 is the relation between the zonal winds (Fig. 2a) and the short period GW variance (Fig. 2b). During 2004-2006, the zonal winds and the GW variance do not show any systematic relation. During 2008-2010, however, there is a systematic relation between the two, with the winds becoming westward during the times of enhanced GW variance. Between 2008 and 2010, the short period GW variance (in Fig. 2b) during the March equinox at $88 \mathrm{~km}$ decreases, with a variance of $115 \mathrm{~m}^{2} \mathrm{~s}^{-2}$ in 2008 to $107 \mathrm{~m}^{2} \mathrm{~s}^{-2}$ in 2009 and $82 \mathrm{~m}^{2} \mathrm{~s}^{-2}$ in 2010. Associated with this decrease, the zonal wind in Fig. 1a (at $88 \mathrm{~km}$ ) becomes less westward with a peak value 
(a) Zonal wind

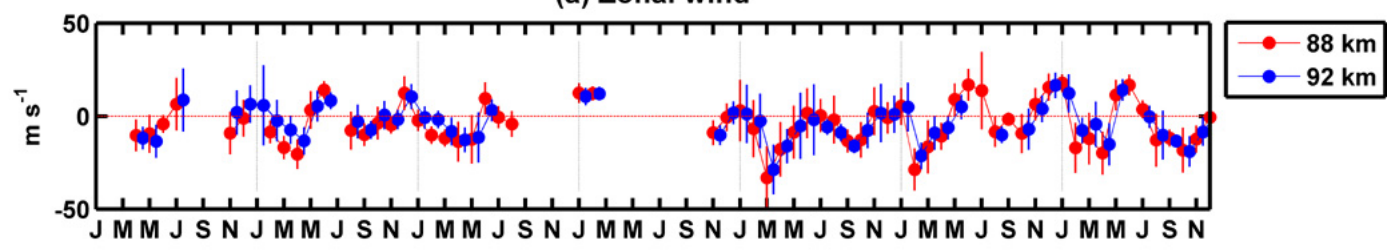

(b) 20-120 $\min \mathrm{GW}$ variance

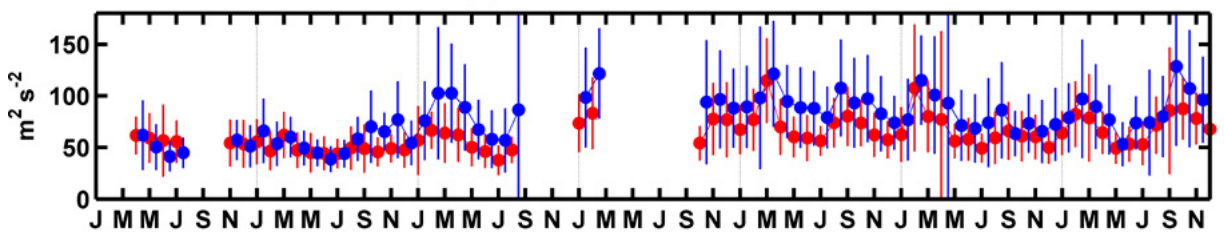

(c) 2-10 hour GW variance

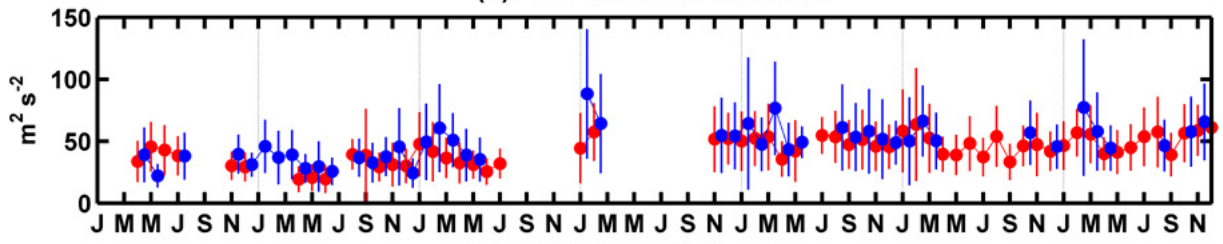

(d) 10-20 hour GW variance

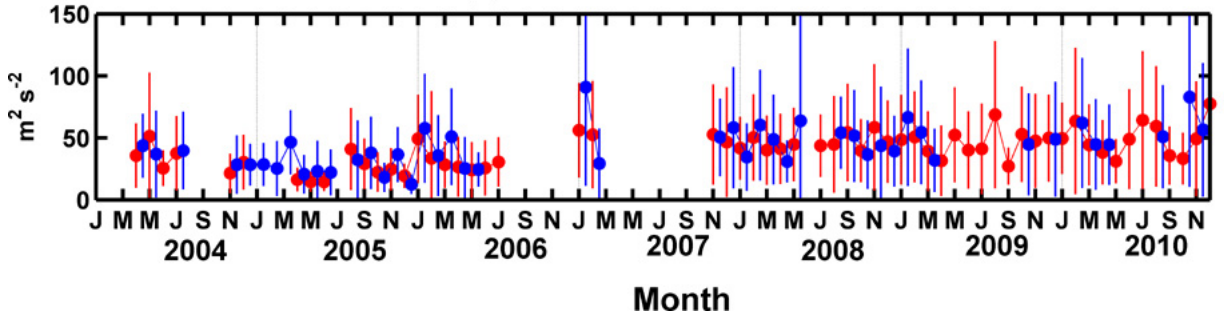

Fig. 2. Variability of the monthly mean (a) zonal wind, (b) 20-120 min gravity wave variance, (c) 2-10 h gravity wave variance, and (d) $10-$ $20 \mathrm{~h}$ gravity wave variance between 2004 and 2010 . The $\mathrm{GW}$ variance is a mean of zonal and meridional variances $\left(\left(u^{\prime 2}+v^{\prime 2}\right) / 2\right)$. The vertical lines represent the standard deviations of monthly mean values. The values at $92 \mathrm{~km}$ are shifted forward by 0.5 of a month.

of $-33 \mathrm{~m} \mathrm{~s}^{-1}$ in 2008 to $-28 \mathrm{~m} \mathrm{~s}^{-1}$ in 2009 and $-19 \mathrm{~m} \mathrm{~s}^{-1}$ in 2010. The annual mean $\mathrm{GW}$ variance at $88 \mathrm{~km}$ is larger in $2008\left(71 \mathrm{~m}^{2} \mathrm{~s}^{-2}\right)$ than in $2009\left(65 \mathrm{~m}^{2} \mathrm{~s}^{-2}\right)$ and 2010 $\left(69 \mathrm{~m}^{2} \mathrm{~s}^{-2}\right)$. Figure $2 \mathrm{a}$ shows that the annual mean zonal winds are more westward in 2008 than in 2009 and 2010. Similar variability is observed at other heights also. Thus, associated with large GW variance in 2008, the winds are westward throughout the year. In 2010, the GW variance in two equinoxes is nearly equal (except at $92 \mathrm{~km}$ ) and the peak westward winds are also nearly equal. Thus, in general, a one-to-one relationship can be noted between the short period GW variance and the zonal winds during 2008-2010, with the winds becoming westward during the times of GW enhancement. On the contrary, such a relationship between the short period GW variance and the zonal winds is not observed during 2004-2006 period. Further, the zonal winds and the GW variance are larger during 2008-2010 than during 2004-2006.

The relationship between the zonal winds and the short period GW variance can be more clearly quantified through a correlative analysis, shown in Fig. 3a and b for 2004-2006 and 2008-2010, respectively. Here, we used the monthly mean values of zonal wind and the GW variance for heights of $88 \mathrm{~km}, 90 \mathrm{~km}$, and $92 \mathrm{~km}$. Note that the correlations mentioned here are significant with $95 \%$ confidence level. During 2004-2006 (Fig. 3a) the overall correlation coefficient $(R)$ is -0.3 and thus not much correlation is observed between the zonal winds and the short period GW variance. However, during 2008-2010 (Fig. 3b) the zonal winds correlate well with the short period GW variance with a correlation coefficient $(R)$ of -0.7 . Furthermore, correlation coefficients $(r)$ between the two are also calculated for each year during 2008-2010 and shown in Fig. 3b. From the straight line in Fig. 3b, we can note that $1 \mathrm{~m} \mathrm{~s}^{-1}$ change in the zonal wind corresponds to about $2.12 \mathrm{~m}^{2} \mathrm{~s}^{-2}$ change in the GW variance. In 2004 and 2006 sufficient data points do not exist to calculate realistic correlation. In 2005 the correlation coefficient is -0.25 . These figures emphasize the fact that the westward winds are associated with enhancement in the GW variance during 2008-2010. The winds are eastward 
(a) 2004-2006

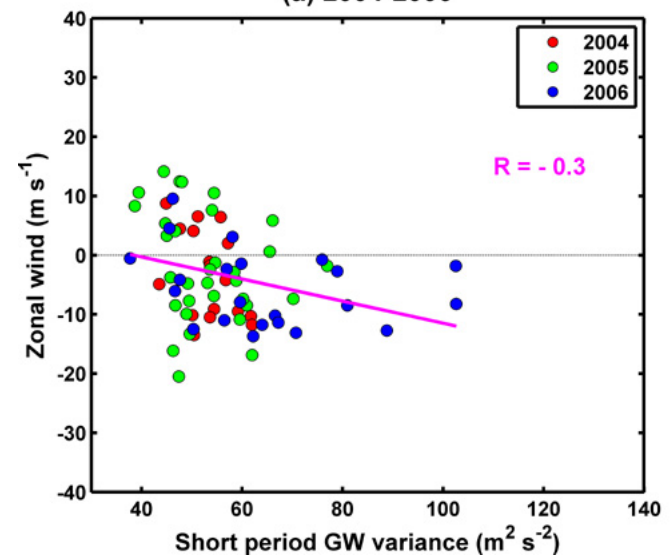

(b) 2008-2010

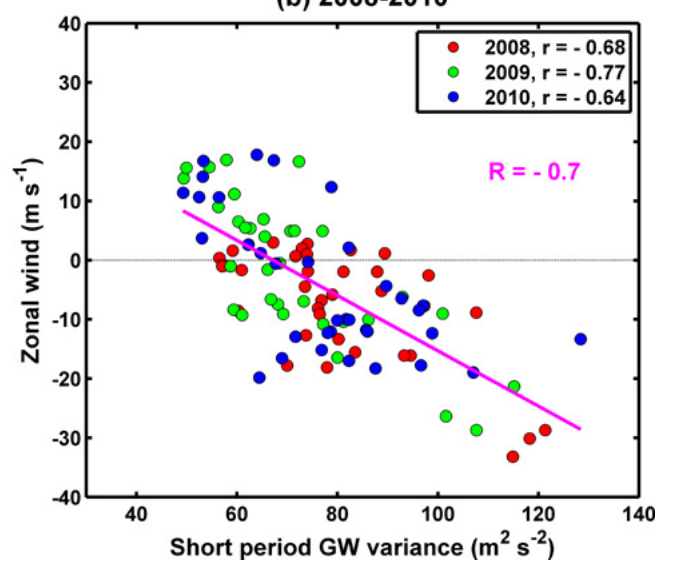

Fig. 3. Scatter plot between zonal winds and the short period gravity wave variance $\left(\left(u^{\prime 2}+v^{\prime 2}\right) / 2\right)$ for (a) 2004-2006, and (b) 2008-2010. Correlation coefficients for individual years $(r)$ and overall correlation coefficients $(R)$ are shown.

(a) 2008
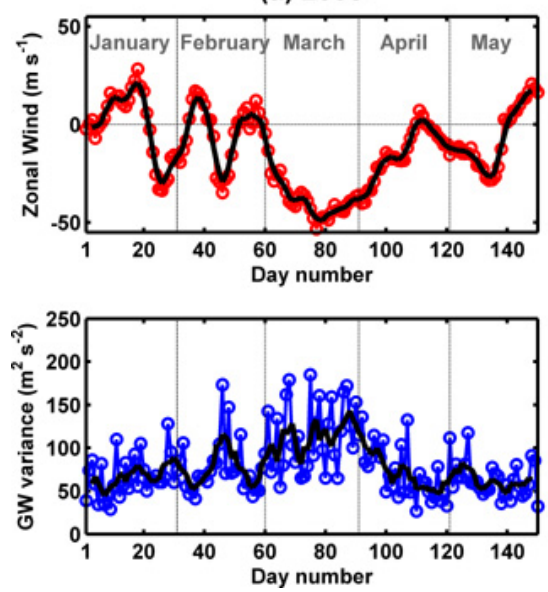

(b) 2009
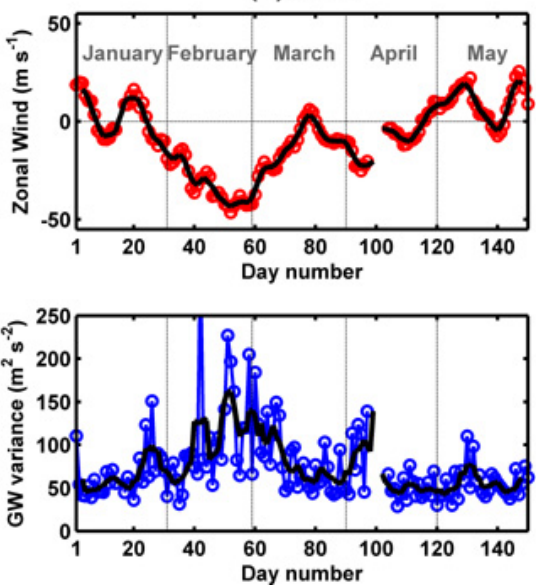

(c) 2010
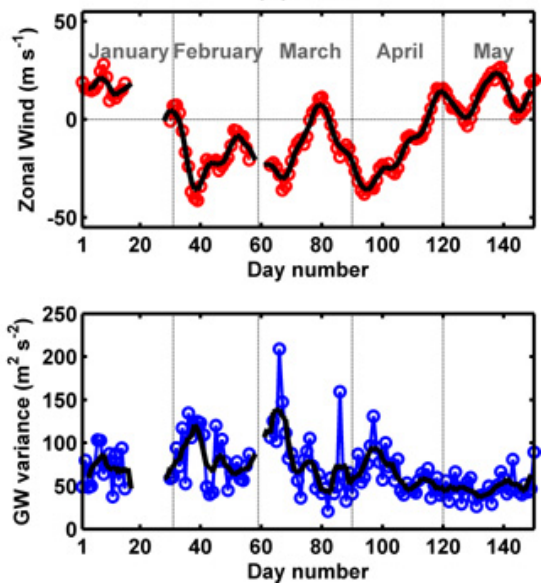

Fig. 4. Variability of the zonal wind (top panels) and short period $(20-120 \mathrm{~min})$ gravity wave variance $\left(\left(u^{\prime 2}+v^{\prime 2}\right) / 2\right)($ bottom panels) in (a) 2008, (b) 2009, and (c) 2010 at $88 \mathrm{~km}$. Thick solid lines correspond to the 5-day running mean.

during the times of less GW activity. Further analysis (not shown here) shows that the zonal winds weakly correlate with the $2-10 \mathrm{~h} \mathrm{GW}$ variance $(R=-0.2$ during $2004-2006$ and $R=-0.3$ during 2008-2010) and they show no significant correlation with $10-20 \mathrm{~h}$ period GW variance. The meridional winds, on the other hand, do not show any significant correlation with GWs in all three bands.

To demonstrate the relation between the zonal winds and the short period (20-120 min) GW variance on a shorter time scale, we show smaller segments of the data in Fig. 4. Figures $4 \mathrm{a}, \mathrm{b}$ and $\mathrm{c}$ show the zonal winds (top panels) and the short period GW variance (20-120 min) (bottom panels) for the first $1-150$ days in 2008, 2009, and 2010, respectively for $88 \mathrm{~km}$. Corresponding months are mentioned in the top panel. The thick solid line in each panel represents 5-day running mean. From these figures, the tendency for winds being westward during the times of enhanced $\mathrm{GW}$ variance is obvious in all three years. Associated with fluctuations at a period of a few days in the zonal wind (intra-seasonal oscillations) (e.g. Isoda et al., 2004), the GW variance also shows fluctuations in nearly similar periods. From Fig. 4, we can also study the relation between the GW variance and the zonal wind on a day-to-day basis. If we consider that the wave-mean flow interaction is taking place, then one would expect the peak in the GW variance to occur before the peak in the westward wind (e.g. near day number 75 in 2008, 51 in 2009, and 66 in 2010). This, however, is not true every time, as sometimes the most extreme values of $\mathrm{GW}$ variance appear in phase with the zonal wind (e.g. near day number 46 in 2008) or lag behind the extrema of zonal wind curve (e.g. near day number 26 in 2008, 95 in 2009, and 94 in 2010). The correlation coefficient $(R)$ between the 5-day running averaged zonal winds and that of GW variance is -0.54 in $2008,-0.53$ in 2009 , and -0.51 in 2010 . The lower values 
of $R$ are possibly due to the fact that the GW variance shows many short-term fluctuations or that the zonal wind is affected by forcing due to residual circulation.

\section{Discussion}

Zonal winds in the equatorial mesopause region over Pameungpeuk show a marked semiannual oscillation, with westward winds during equinoxes and eastward winds during solstices. In 2005, 2008, and 2009, it is clearly observed that the first westward cycle is stronger than the second one. These results are in agreement with previous observations (Garcia et al., 1997; Sridharan et al., 2007). In 2010, however, the amplitude of two westward cycles is nearly equal. The present observations also illustrate that in 2008 the zonal winds show an anomalous behavior, in the sense that the winds are westward throughout the year (annual mean $-7 \mathrm{~m} \mathrm{~s}^{-1}$ ).

Previous studies have shown that the enhanced westward wind during March equinox was repeated, with an interval of 1-3 years with considerable inter-annual variability. During 1993, 1995 and 1997, this interval was found to be similar to that of stratospheric QBO (SQBO) and referred to as the mesospheric QBO (MQBO) (Burrage et al., 1996; Baldwin et al., 2001; Sridharan et al., 2007; Ratnam et al., 2008). However, this so-called MQBO was not observed for several years after 2002 (Sridharan et al., 2007). In the present study also, the large enhancement in the westward winds is observed only during 2008 and 2009.

The short period (20-120 min) GW variance also shows a clear semiannual variability with peak variance during equinoxes. While the first peak is stronger than the second in 2008 and 2009, the second peak is slightly stronger than the first in 2010 . The longer $(2-10 \mathrm{~h}, 10-20 \mathrm{~h})$ period $\mathrm{GW}$ variance, on the other hand, does not show pronounced seasonal variability. This can be explained considering that the short period GWs have large phase velocities compared to the long period ones (Fritts and Alexander, 2003) and hence they are less susceptible to the critical level filtering by the underlying wind systems and can easily reach MLT heights. Previous observations from low-latitudes also showed a SAO of the GW activity, with enhancements during equinoxes (Antonita et al., 2008; Clemesha et al., 2009).

The most important result in this study is the correlation between the zonal winds and the short period GW variance during 2008-2010, with winds becoming westward during the times of enhanced GW variance. Although the GW variance alone cannot tell whether wave-mean flow interaction is taking place or not (which requires divergence of momentum flux), here we try to understand what causes such a good correlation between the two, considering that the GWs are contributing to the MSAO. Alexander et al. (2008) have shown that the GW variance in the troposphere has a seasonal variation, with the variance being more during equinoxes and less during solstices. Thus, more GWs are generated during equinoxes and less during solstices. We also presume that the GWs are always generated in pairs, with eastward and westward propagations. These GWs, while propagating upward, undergo critical level filtering at various levels of the atmosphere. Due to the weak westward winds throughout the year in the tropical troposphere over Indonesian sector, only the westward propagating GWs that have phase speeds greater than the tropospheric winds and all the eastward propagating GWs reach the SSAO heights. The SSAO is characterized by eastward winds during equinoxes and westward winds during solstices (e.g. Dunkerton, 1982; Garcia et al., 1997). Hence, during equinoxes (solstices) the eastward (westward) propagating GWs will be filtered by the SSAO and hence more westward (eastward) propagating GWs will reach the MLT region. Since, at the source region, more GWs are generated during equinoxes and less during solstices, the westward propagating GWs that reach the MLT region during the equinoxes dominate over the eastward propagating GWs during solstices. Considering that these westward propagating GWs contribute westward momentum to the zonal wind, the correlation between the zonal winds and the GWs and large westward winds during equinoxes (shown in Fig. 2a) can be understood to some extent. Further, filtering effect by the SQBO and altitudinal shift of MSAO may lead to interannual variability of the westward winds.

There exist some studies on the relation between the zonal winds at a single location with those at other location of similar latitude or those of zonal mean zonal winds (Isoda et al., 2004; Xu et al., 2009). Isoda et al. (2004) studied the intraseasonal oscillation (ISO) of the zonal wind in the MLT region with periods of $20-100$ days at Pontianak $\left(0^{\circ} \mathrm{N}\right.$, $\left.109^{\circ} \mathrm{E}\right)$ and Christmas Island $\left(2^{\circ} \mathrm{N}, 157^{\circ} \mathrm{W}\right)$ and found that the phase of the ISO is nearly similar at the two sites, in spite of $90^{\circ}$ separation between them. Xu et al. (2009) compared the time mean zonal winds observed by a meteor radar on Maui $\left(20.75^{\circ} \mathrm{N}, 156.43^{\circ} \mathrm{W}\right)$ with the zonalmean zonal winds at $20^{\circ} \mathrm{N}$ observed by the Sounding of the Atmosphere satellite using Broadband Emission Radiometry (SABER) instrument and found good correlation between the two. These studies suggest that the zonal winds observed at a single location are a close approximation of the zonal-mean zonal winds. GW activity measurements in the stratosphere, on the other hand, showed considerable longitudinal variability (Tsuda et al., 2000). Accordingly, we anticipate that the GW activity in the MLT region also shows longitudinal variability. Thus, the GW variance in the present study corresponds to a single location, whereas the zonal winds above Pameungpeuk are considered to approximate the zonal-mean zonal winds. Even so, the close correlation between the zonal winds and the GW variance is striking. Accordingly, the explanation given in the previous paragraph requires that the GW activity in the Indonesian region is the main driver of the (zonal mean) zonal winds. However, it is difficult to imagine that the GWs generated in a limited area are driving 
the zonal-mean zonal winds. Interestingly, there is no such relationship between the winds and the GW variance during 2004-2006 period. Along with the short period GWs, the other possible drivers such as intermediate-scale Kelvin and inertia GWs, meridional advection, diurnal tide deposition, and GW-tidal interactions and their relative contributions need to be considered.

Modeling studies in the troposphere showed that short spatial and temporal scales of the westward propagating GWs are generated more compared to the eastward propagating GWs (Horinouchi and Yoden, 1998; Kawatani et al., 2010). Thus, temporal and spatial scales of the GWs interacting with background winds in the MSAO region may be smaller in the westward wind shear phase, leading to larger westward winds during the times of short period GW enhancement. In other words, there could be eastward forcing from longer period and longer horizontal wavelength GWs during solstices. Thus, we anticipate that there are more short period GWs during the westward phase of the wind. The large GW variance during the equinoxes in the short period band (in Fig. 2b) and reduction in the seasonal contrast with increasing GW period (in Fig. 2c and d) support this assumption.

\section{Conclusions}

Zonal winds in the equatorial MLT region over Pameungpeuk $\left(7.4^{\circ} \mathrm{S}, 107.4^{\circ} \mathrm{E}\right)$ show a distinct semiannual oscillation, with westward winds during equinoxes and eastward winds during solstices. The variance associated with the short period (20-120 min) GWs, which is considered as a proxy for GW activity, also shows semiannual variability, with enhancements during equinoxes. The most important result in this study is the close relationship between the zonal winds and the short period GW variance, with the winds being westward during the times of enhanced GW activity during 2008-2010. This relationship exists on monthly mean values and daily values as well. For monthly mean values, $1 \mathrm{~m} \mathrm{~s}^{-1}$ change in the zonal wind corresponds to about $2.12 \mathrm{~m}^{2} \mathrm{~s}^{-2}$ change in the GW variance. The correlation between the zonal winds and the short period GW variance is less during 2004-2006 than during 2008-2010. The variance associated with longer period (2-10 $\mathrm{h}$ and $10-20 \mathrm{~h}) \mathrm{GWs}$, on the other hand, does not show much seasonal variation and hence does not correlate well with zonal winds.

Thus, the correlation between the zonal winds and the short period GW variance in the present paper is impressive and its clear understanding requires various experiments with multiple instruments and long-term observations. Though many questions remain about what causes such a remarkable correlation between the two, our results open a new perspective for investigating the global relation between zonal winds and GWs. Furthermore, these results will also help to improve the general circulation models, by incorporating the contribution from the short period GWs to better reproduce the observed MSAO.

Acknowledgements. The work of N. Venkateswara Rao was supported by the Japan Society for the Promotion of Science (JSPS) foundation (ID No: P09232). We thank S. Miyahara for his helpful comments on the original manuscript. We also thank Dennis Riggin for helpful discussions and careful reading of the revised manuscript. The Pameungpeuk MF radar is operated as a collaborative project between RISH and LAPAN. This study was supported in part by the Japanese Ministry of Education, Culture, Sports, Science and Technology (MEXT) through Grants-in-Aid for Scientific Research (19403009 and 22253006).

Topical Editor C. Jacobi thanks one anonymous referee for her/his help in evaluating this paper.

\section{References}

Alexander, S. P., Tsuda, T., Shibagaki, Y., and Kozu, T.: Seasonal gravity wave activity observed with the Equatorial Atmosphere Radar and its relation to rainfall information from the Tropical Rainfall Measuring Mission, J. Geophys. Res., 113, D02104, doi:10.1029/2007JD008777, 2008.

Antonita, T. M., Ramkumar, G., Kumar, K. K., and Deepa, V.: Meteor wind radar observations of gravity wave momentum fluxes and their forcing toward the Mesospheric Semiannual Oscillation, J. Geophys. Res., 113, D10115, doi:10.1029/2007JD009089, 2008.

Baldwin, M. P., Gray, L. J., Dunkerton, T. J., Hamilton, K., Haynes, P. H., Randel, W. J., Holton, J. R., Alexander, M. J., Hirota, I., Horinouchi, T., Jones, D. B. A., Kinnersley, J. S., Marquardt, C., Sato, K., and Takahashi, M.: The quasi-biennial oscillation, Rev. Geophys., 39, 179-229, 2001.

Burrage, M. D., Vincent, R. A., Mayr, H. G., Skinner, W. R., Arnold, N. F., and Hays, P. B.: Long-term variability in the equatorial middle atmosphere zonal wind, J. Geophys. Res., 101, 12847-12854, doi:10.1029/96JD00575, 1996.

Clemesha, B. R., Batista, P. P., Buriti da Costa, R. A., and Schuch, N.: Seasonal variations in gravity wave activity at three locations in Brazil, Ann. Geophys., 27, 1059-1065, doi:10.5194/angeo27-1059-2009, 2009.

Dunkerton, T. J.: Theory of the Mesopause Semiannual Oscillation, J. Atmos. Sci., 39, 2681-2690, 1982.

Fritts, D. C. and Alexander, M. J.: Gravity wave dynamics and effects in the middle atmosphere, Rev. Geophys., 41, 1003, doi:10.1029/2001RG000106, 2003.

Garcia, R., Dunkerton, T., Lieberman, R., and Vincent, R.: Climatology of the semiannual oscillation of the tropical middle atmosphere, J. Geophys. Res., 102, 26019-26032, 1997.

Gurubaran, S. and Rajaram, R.: Mean winds, tides, and gravity waves during the westward phase of the mesopause semiannual oscillation (MSAO), J. Geophys. Res., 106, 31817-31824, 2001.

Hirota, I.: Equatorial Waves in the Upper Stratosphere and Mesosphere in Relation to the Semiannual Oscillation of the Zonal Wind, J. Atmos. Sci., 35, 714-722, 1978.

Hoffmann, P., Rapp, M., Singer, W., and Keuer, D.: Trends of mesospheric gravity waves at northern middle latitudes during summer, J. Geophys. Res., 116, D00P08, doi:10.1029/2011JD015717, 2011. 
Horinouchi, T. and Yoden, S.: Wave-mean flow interaction associated with a QBO-like oscillation simulated in a simplified GCM, J. Atmos. Sci., 55, 502-526, 1998.

Isoda, F., Tsuda, T., Nakamura, T., Vincent, R. A., Reid, I. M., Achmad, E., Sadewo, A., and Nuryanto, A.: Intraseasonal oscillations of the zonal wind near the mesopause observed with medium-frequency and meteor radars in the tropics, J. Geophys. Res., 109, D21108, doi:10.1029/2003JD003378, 2004.

Kawatani, Y., Watanabe, S., Sato, K., Dunkerton, T. J., Miyahara, S., Takahashi, M.: The Roles of Equatorial Trapped Waves and Internal Inertia-Gravity Waves in Driving the Quasi-Biennial Oscillation. Part I: Zonal Mean Wave Forcing, J. Atmos. Sci., 67, 963-980, doi:10.1175/2009JAS3222.1, 2010.

Lieberman, R. S. and Hays, P. B.: An Estimate of the Momentum Deposition in the Lower Thermosphere by the Observed Diurnal Tide, J. Atmos. Sci., 51, 3094-3105, 1994.

Offermann, D., Wintel, J., Kalicinsky, C., Knieling, P., Koppmann, R., and Steinbrecht, W.: Long-term development of short-period gravity waves in middle Europe, J. Geophys. Res., 116, D00P07, doi:10.1029/2010JD015544, 2011

Ratnam, M. V., Kumar, G. K., Murthy, B. V. K., Patra, A. K., Rao, V. V. M. J., Rao, S. V. B., Kumar, K. K., and Ramkumar, G.: Long-term variability of the low latitude mesospheric SAO and QBO and their relation with stratospheric QBO, Geophys. Res. Lett., 35, L21809, doi:10.1029/2008GL035390, 2008.

Richter, J. H. and Garcia, R. R.: On the forcing of the Mesospheric Semi-Annual Oscillation in the Whole Atmosphere Community Climate Model, Geophys. Res. Lett., 33, L01806, doi:10.1029/2005GL024378, 2006.

Sassi, F. and Garcia, R. R.: The Role of Equatorial Waves Forced by Convection in the Tropical Semiannual Oscillation, J. Atmos. Sci., 54, 1925-1942, 1997.
Sridharan, S., Tsuda, T., and Gurubaran, S.: Radar observations of long-term variability of mesosphere and lower thermosphere winds over Tirunelveli $\left(8.7^{\circ} \mathrm{N}, 77.8^{\circ} \mathrm{E}\right)$, J. Geophys. Res., 112, D23105, doi:10.1029/2007JD008669, 2007.

Taori, A., Patra, A. K., and Joshi, L. M.: Gravity wave seeding of equatorial plasma bubbles: An investigation with simultaneous $F$ region, $E$ region, and middle atmospheric measurements, J. Geophys. Res., 116, A05310, doi:10.1029/2010JA016229, 2011.

Tsuda, T., Nishida, M., Rocken, C., and Ware, R.: A Global Morphology of Gravity Wave Activity in the Stratosphere Revealed by the GPS Occultation Data (GPS/MET), J. Geophys. Res., 105, 7257-7273, 2000.

Venkateswara Rao, N., Patra, A. K., and Rao, S. V. B.: Some new aspects of low-latitude $E$-region QP echoes revealed by Gadanki radar: Are they due to Kelvin-Helmholtz instability or gravity waves?, J. Geophys. Res., 113, A03309, doi:10.1029/2007JA012574, 2008.

Venkateswara Rao, N., Shibagaki, Y., and Tsuda, T.: Diurnal variation of short-period (20-120 min) gravity waves in the equatorial Mesosphere and Lower Thermosphere and its relation to deep tropical convection, Ann. Geophys., 29, 623-629, doi:10.5194/angeo-29-623-2011, 2011a.

Venkateswara Rao, N., Tsuda, T., Gurubaran, S., Miyoshi, Y., and Fujiwara, H.: On the occurrence and variability of the terdiurnal tide in the equatorial mesosphere and lower thermosphere and a comparison with the Kyushu-GCM, J. Geophys. Res., 116, D02117, doi:10.1029/2010JD014529, 2011b.

Xu, J., Smith, A. K., Liu, H.-L., Yuan, W., Wu, Q., Jiang, G., Mlynczak, M. G., Russell, J. M., and Franke, S. J.: Seasonal and quasi-biennial variations in the migrating diurnal tide observed by Thermosphere, Ionosphere, Mesosphere, Energetics and Dynamics (TIMED), J. Geophys. Res., 114, D13107, doi:10.1029/2008JD011298, 2009. 